\title{
Improving Outcome for the Elderly Surgical Patients in a Singapore Teaching Hospital
}

\author{
Si Ching Lim*, Peter CL Chow, Fuyin Li, Swee Sim Hiew, Lau Soy Soy and Zhang Di \\ Department of Geriatric Medicine, Singapore \\ *Corresponding author: Si Ching Lim, Department of Geriatric Medicine, Singapore
}

Submission: December 24, 2018; Published: January 11, 2019

\begin{abstract}
The elderly patients admitted under surgery have longer lengths of stay and develop multiple complications during their hospital stay particularly with delirium, medical complications and functional decline. A Geriatrician's input was helpful to identify incident and postop delirium early and put in measures to improve outcome, together with better nursing care and pharmacist's input to reduce harm from medications.
\end{abstract}

Keywords: Elderly; Surgical patients; Postoperative delirium

\section{Introduction}

Singapore is rapidly ageing, and the elderly admitted under Department of General Surgery for both elective surgeries as well as emergency surgeries will likely increase over the years. The hospitalized elderly is vulnerable to develop complications associated with hospital stay, particularly iatrogenic complications such as, delirium, restraint use, falls, malnutrition and functional decline. These adverse effects may occur immediately at admission, if due diligent care is not put in place [1]. Among the elderly patients admitted for surgical care, perioperative complications are directly associated with poor outcome and the risk increases with advancing age. Perioperative complications among those over 80 have a $25 \%$ greater 30 day mortality, compared to the uncomplicated elderly [2,3]. Initiatives to improve overall care of the elderly under surgical care were considered and with approval from senior management, a new pilot service was developed.

\section{Project aims}

A. Early identification of incident or postoperative delirium, by the surgical nursing and medical staffs followed by referrals to the geriatricians and pharmacist.

B. Early mobilization and referrals for rehabilitation.

C. Educate general surgical ward nurses on principles of geriatric nursing particularly on management of agitated elderly and reduce physical restraint usage.

D. Review the results of pilot project to further improve outcome of the elderly surgical patients.

\section{Methodology}

This General Surgery- Geriatric Medicine collaboration is currently ongoing in a 1000 bedded teaching hospital in Singapore. A team consisting of two geriatricians, Advanced Practice Nurses
(APN), senior nursing from the general surgical wards, surgical ICU and a pharmacist with an interest in pharmacology for the elderly was formed. The team is led by a senior Geriatrician. From the outset, it was felt that there is a need to improve the background knowledge of delirium, postop delirium, overall care of the elderly patients especially those who are agitated and restless in the wards. Lectures and bedside tutorials were put in place simultaneously to improve nurses' knowledge and confidence in managing the elderly patients with delirium.

\section{Didactic lectures on clinical diagnosis of postop delirium}

As an introduction to this project, a series of Phase 1 didactic classroom lectures involving a geriatrician and the APN was given as a foundation. The topics covered were, signs and symptoms of delirium, postop delirium, diagnostic tests and management strategies for postop delirium. The APN covered screening for presence of delirium using the Confusion Assessment Method (CAM) and basic Gerontological nursing care for a delirious elderly in a general surgical ward. This was followed by a two-day workshop for the more senior surgical ward nurses, so they can continue to teach their new and junior staffs. A total of 264 registered nurses from the surgical wards attended Phase 1 lectures.

\section{Pilot project in identification of postop delirium}

The whole department of General Surgeons were briefed for the purpose of this new initiative with the aim for early physician's input and to lower hospital associated complications. There were ad hoc meetings between the geriatricians and a representative from the department of general surgery for feedback and information sharing.

The nurses in the General Surgical wards and Surgical ICU were able to screen for delirium using CAM, since the Phase 1 lectures 
started. Once the patients screened positive for delirium, the surgical team triggers a referral to the Geriatrician. Data collection was done over a period of 4 months between April 2016 and July 2016. Individual discharge summaries were reviewed by the geriatricians and data collected was reviewed.

\section{Concurrent bedside tutorial for management of elderly with challenging behavior}

It was felt that didactic lectures would be more productive if the nurses had hands on teaching on non-pharmacological, individualized management of the elderly who were agitated and restless in the general wards. Once weekly nurse led bedside tutorials were initiated in the general surgical wards from April 2016. The cases chosen for teaching focused on the elderly who were either delirious or have behavioral symptoms of dementia where many of them required physical restraints to contain their behavioral symptoms.

\section{Lectures on management of elderly with challenging behaviors}

The team met again after all the nurses have attended Phase 1 lectures to gather feedback and reviewed nurses' requests for lecture topics for Phase 2 of lectures. Most nurses felt powerless handling the agitated and restless elderly, and most of them resort to application of restraints for patient safety. As a result, Phase 2 lectures focused on non-pharmacological management of challenging behavior, which included causes for challenging

Table 1: Postoperative delirium. behavior, modifications of environment, reminiscence therapy, activities like art and craft and the APN lectured with case illustrations. The total attendance for these lectures was 299 nurses. An anonymous survey form was handed out after the completion of phase 2 lecture series for feedback. The forms were given out for the nurses in the general surgical wards to fill in for one whole day.

\section{Service expanded to involve all referrals from general surgery to geriatric medicine}

At 4 months, the service was reviewed by the Geriatricians and the General Surgeons and was felt that the physician's input was beneficial to the elderly surgical delirious patients. The collaboration was extended to include all referrals from the department of General Surgery, from July 2016 until present. The patient data from the last 6 months of 2016 were collected and analyzed. The hospital's data bank was approached to release data for physical restraint usage for these surgical wards. Service will soon be up for review for the next phase of development.

\section{Results}

\section{Postop delirium}

There was a total of 27 patients referred for postoperative delirium, between April to June 2016. The patients were either taken over by the Geriatricians or reviewed regularly until they were medically stable. The mean length of stay was 28days (595days). There were 6 patients who had recurrent admission in 30days. Results is as shown in Table 1.

\begin{tabular}{|c|c|}
\hline Gender & Male $=17$, Female $=10$ \\
\hline Age range & Mean 82 (66-94) \\
\hline Type of surgery & $\begin{array}{c}\text { Hepatobiliary }+ \text { Pancreas }=9 \\
\text { Upper GI }=7 \\
\text { Colorectal }=5 \\
\text { Vascular }=3 \\
\text { Others- general surgery }=2 \text {, Ortho= } 1\end{array}$ \\
\hline Onset of delirium (from postop day) & $\begin{array}{c}0=2 \\
\text { Day } 1=17 \\
\text { Day } 2-4=4 \\
\text { Day } 10 \text { and beyond }=4\end{array}$ \\
\hline Electrolyte abnormalities & $\begin{array}{c}\text { Hyponatraemia }=11 \\
\text { Hyperglycaemia }=6 \\
\text { AKI }=6 \\
\text { Acidosis }=4 \\
\text { Others= hypokalaemia, hypocalaemia, hypo PO4 } \\
\text { No electrolyte abnormalities }=3\end{array}$ \\
\hline
\end{tabular}




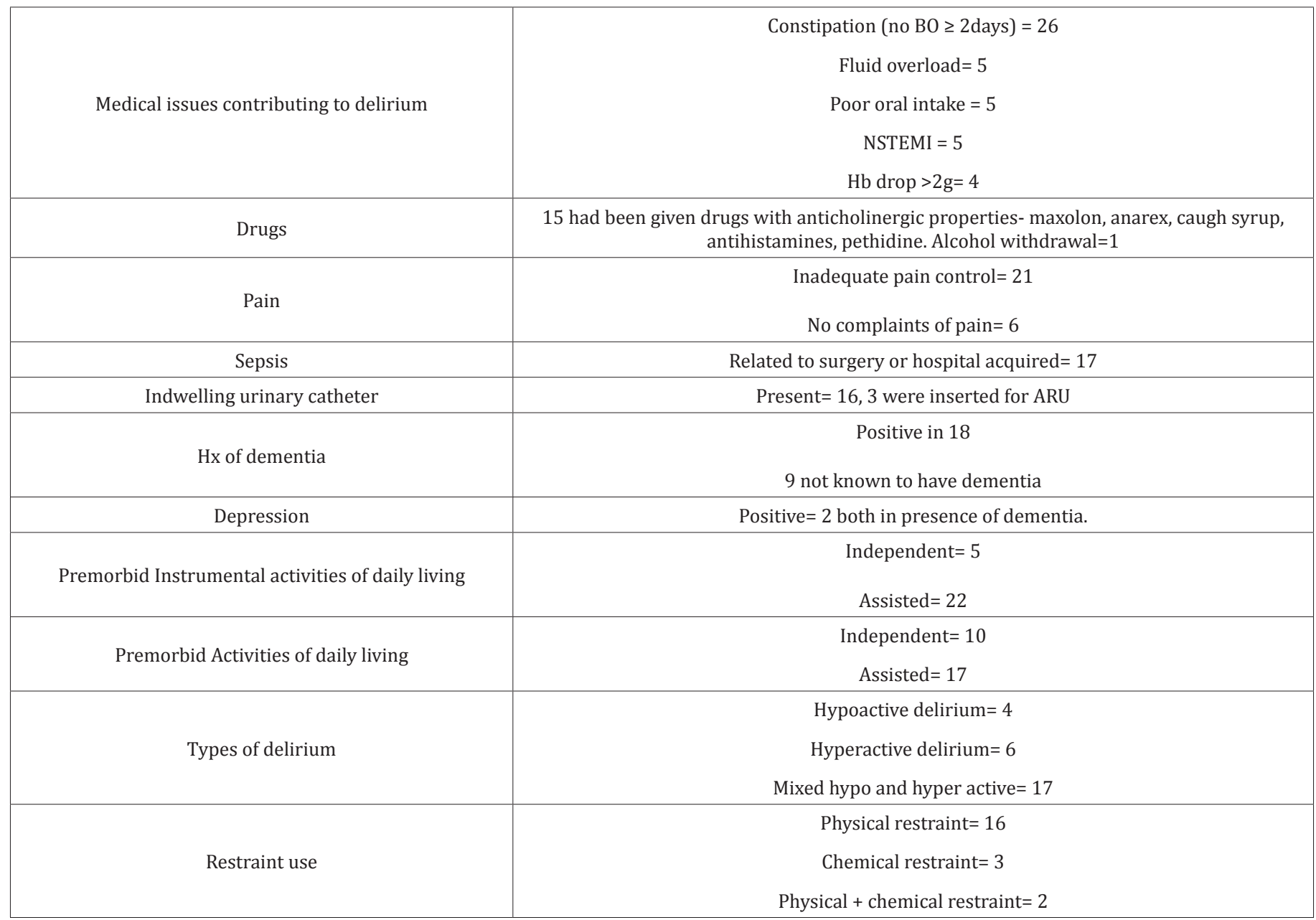

\section{Survey results on Surgical ward nurses after 2 nd series of lectures}

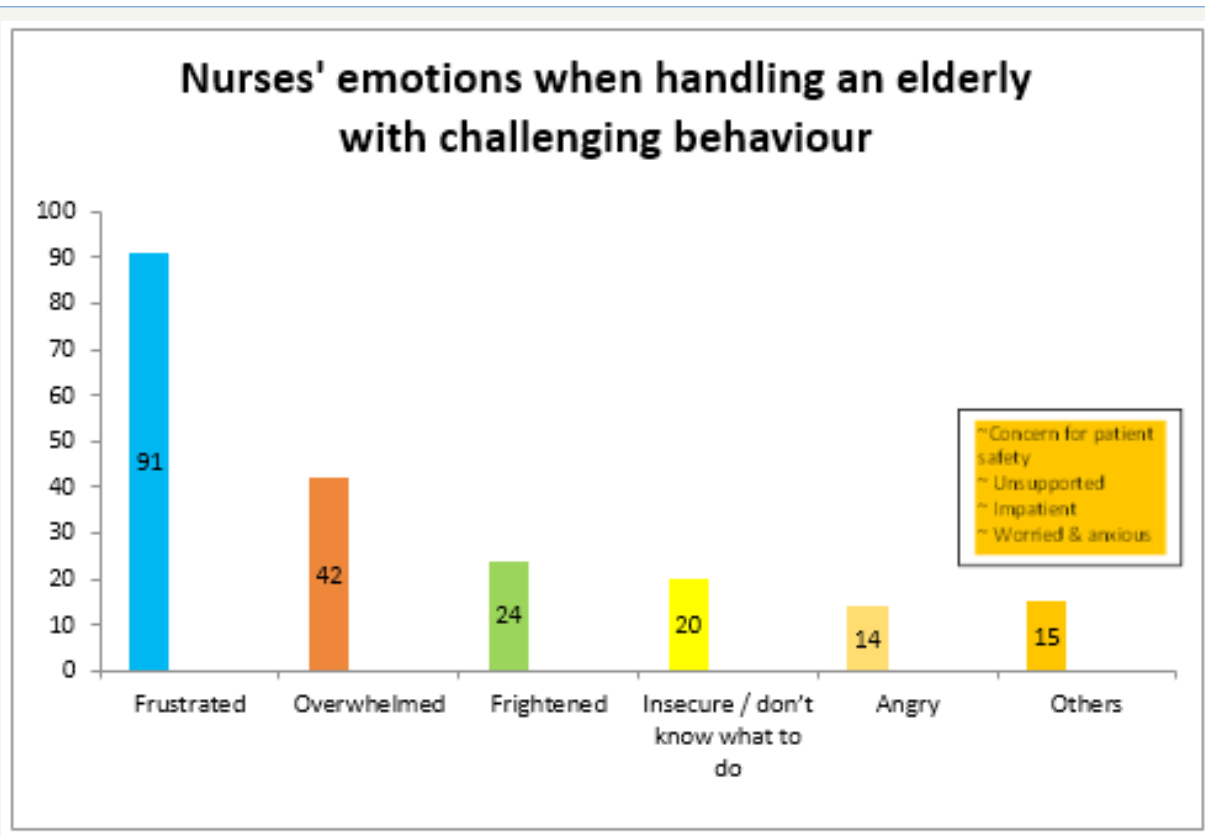

Figure 1

An anonymous survey form was handed out to the nurses in the general surgical wards to collect feedback on the 2 series of lectures they have attended. It was a point prevalence data collection on one full working day. There were 137 survey forms filled and returned. The working experience of these nurse's ranges from 4 months to 51 years, with a mean of 6 years. Majority (88\%) of 
the nurses found the information given was relevant to their day to day work. The emotional reactions while handling elderly with challenging behavior is shown in Figure 1. Having attended the series of tutorials, $57 \%$ felt more confident handling elderly with challenging behavior. Most of them (81\%) learned that there are causes like pain, hunger, thirst, fatigue, boredom etc. which can give rise to challenging behavior, and $89 \%$ of nurses will look for these possible causes when they have an agitated patient under their care. Even though majority of nurse (69\%) believed restraints cause less falls and only $8 \%$ believed the contrary, $79 \%$ of nurses will avoid putting on a physical restraint as the first line of management after the lectures.

\section{Referrals from General surgery to the department of geriatric medicine July-December 2016}

A total of 76 patients were referred to the Geriatricians in the last 6 months of 2016. There were 36 male and 39 female patients. The mean age was 81 (range 66-97). The mean length of stay was 24 days (range 3-138 days). There were 42 patients (55\%) who did not undergo any surgical procedures. The commonest reasons for referrals included physical function decline, multiple medical problems needing stabilization, poor feeding, postoperative or new onset delirium and social issues. There were 39 (51\%) patients with a background history of dementia. The geriatricians took over $44(68 \%)$ patients to their inpatient beds for further management. There were 35 (46\%) patients who had feeding issues during their stay and were all referred to the dietitians for oral nutritional supplements. Most patients 44 (58\%) were discharged back to their own home. There were 22 patients (29\%) who were discharged to community step down care facilities for further rehabilitation. There were 7 patients discharged to nursing homes and 4 deaths.

\section{Hospital restraint usage pre and post intervention}

The hospital's data for restraint usage in the wards is a point prevalence. The number of patients restrained is divided by the total number of patients in the ward, in percentage. The first quarter's data is used as the pre-intervention to compare against the last quarter of 2016 as post intervention data. The tutorials occurred during the $2^{\text {nd }}$ and $3^{\text {rd }}$ quarter of 2016 with bedside tutorials still ongoing. The results are show in in Table 2.

Table 2: Hospital restraint usage.

\begin{tabular}{|c|c|c|c|c|}
\hline Wards & $\mathbf{1}^{\text {st }} \mathbf{Q u a r t e r ~ 2 0 1 6}$ & $\mathbf{2}^{\text {nd }} \mathbf{Q u a r t e r ~ 2 0 1 6}$ & $\mathbf{3}^{\text {rd }} \mathbf{Q u a r t e r ~ 2 0 1 6}$ & $\mathbf{4}^{\text {th }} \mathbf{Q u a r t e r ~ 2 0 1 6}$ \\
\hline A & $0.00 \%$ & $0.00 \%$ & $0.00 \%$ & $0.00 \%$ \\
\hline B & $0.00 \%$ & $0.00 \%$ & $2.78 \%$ & $0.00 \%$ \\
\hline C & $5.71 \%$ & $0.00 \%$ & $2.78 \%$ Ta & $0.00 \%$ \\
\hline D & $0.00 \%$ & $7.14 \%$ & $2.38 \%$ & $2.50 \%$ \\
\hline
\end{tabular}

\section{Discussion}

Delirium can be present in up to $50 \%$ of the postop patients in the surgical wards. The commonest predisposing factors for delirium include advanced age, background history of dementia, previous delirium, pre-op functional dependence, drugs, sensory impairment and multiple comorbidities. There is the vulnerability factor to consider why delirium develops in certain elderly $[4,5]$. The onset is typically within day 1-3 postop, later onset delirium is often associated with other complications like alcohol withdrawal or drugs [6]. In our cohort of patients with postoperative delirium, $23(85 \%)$ developed delirium within 4 days postop and only 4 (15\%) developed delirium after day 10 postop. There were 18 (67\%) patients with prior history of cognitive impairment, most of whom were not previously diagnosed. Most of the patients with postop delirium needed assistance with their activities of daily living $(62 \%)$ and instrumental activities of daily living (81\%) prior to admission.

Most of the elderly in the cohort with postop delirium had electrolyte abnormalities with hyponatremia being the commonest. Acute kidney injury associated with acidosis and potassium abnormalities were also common. Fluid resuscitation often resulted in fluid overload during the subsequent hospital stay. Drugs have been associated with delirium either as a direct toxic effect or drug-drug interactions or indirectly causing organ dysfunction [7]. We found a high percentage of delirious patients were prescribed medications which may have contributed to onset of delirium, particularly drugs with anticholinergic side effects and opioids like Pethidine or a combined prescription of multiple opioids. Pethidine is on the Beer's list as an unsuitable drug for the elderly, particularly elderly with renal impairment because of the long half-life [7].

Delirium is associated with psychomotor changes, with mixed hypo and hyper active delirium being the commonest. Hypoactive delirium is often unrecognized [4]. In our cohort, mixed delirium is observed among $62 \%$ of the elderly postop patients. Physical restraints are often used in the management of elderly with challenging behavior without much evidence to support its benefit. Most of the physical restraints were applied for patient safety reason like falls prevention. Most of the literature on restraint use showed negative feeling on application of restraints among the caregivers but when in doubt, restraints are often applied for the sake of patients' safety [8]. In our cohort of delirious patients, there were $21(78 \%)$ patients on physical, chemical or combination of physical and chemical restraints.

Anothergoalof this project wasto educatenurses on management of challenging behaviors among the elderly who were delirious with background history of dementia with behavioral symptoms. The surgical ward nurses expressed feelings of frustration, being overwhelmed, feeling insecure, angry and impatience towards the elderly with challenging behavior under their care. However, with the education initiatives and encouragement, the nurses' confidence and realization that challenging behaviors may indicate a failure in communicating underlying unmet needs improved. They were also empowered and trained to manage agitation with 
behavioral and environmental modifications. The hospital collects data on quarterly basis on the usage of physical restraints and comparing the restraint data on pre and post intervention, there is a reduction on usage in physical restraints among the 4 surgical wards, even though this is only data collected in the last quarter of 2016. Continual monitoring of restraint usage will be interesting to monitor the success of the programmed.

The data on the overall referrals from the department of surgery suggests that the main causes for referrals are functional decline due to bed rest and hospitalization, wound care and stabilization of medical problems in the postop stay, among which nutrition seem to play an important role. Most of these elderly surgical patients stayed for over 3 weeks, with about $30 \%$ being discharged to step down care for further rehabilitation. There are limitations to this pilot project. The survey forms were only filled in by about a third of the total number of nurses who attended the 2 series of didactic lectures and may not be the overall representation. The post intervention restraint data which we have presented involves only one quarter of the year and longer periods of monitor is required to test the real commitment and effectiveness of the intervention.

\section{Conclusion}

The surgical patients referred to the GS-Geri service are vulnerable to develop hospital associated complications and most of them had very long stay in the hospital due to various complications arising during their stay. The next step is to identify the more frail and vulnerable patients preoperatively and put in measures to reduce hospital associated complications, particularly identifying those at risk of postop delirium, dementia or prior history of delirium, functional decline and undernutrition for early intervention in order to reduce length of stay and improve outcome.

\section{References}

1. Walsh KA (2007) Review: Hospitalization and the elderly. Ann Long Term Care 15: 18-23.

2. Frederick ES (2011) Preventing postoperative complications in the elderly. Anaesthesiol Clin 29(1): 83-97.

3. Hamel MB, Henderson WG, Khuri SF, Daley J (2005) Surgical outcomes for patients aged 80 and older: Morbidity and mortality from major noncardiac surgery. J Am Geriatr Soc 53(3): 424-429.

4. Inouye SK (2006) Delirium in older persons. N Engl J Med 354: 11571165.

5. Litaker D, Locala J, Franco K, Bronson DL, Tannous Z (2001) preoperative risk factors for postoperative delirium. Gen Hosp Psych 23(2): 84-89.

6. Marcantonio ER, Goldman L, Mangione CM, Ludwig LE, Muraca B, et al. (1994) A clinical prediction rule for delirium after elective noncardiac surgery. JAMA 271(2): 134-139.

7. Britton ME (2011) Drugs, Delirium, and older people. J Pharm Pract Res 41: 233-238.

8. SC Lim (2017) Restraint use in the management of dementia in acute hospital setting. Internal Med Res Open J 1(2): 1-4.
Creative Commons Attribution 4.0

International License

For possible submissions Click Here

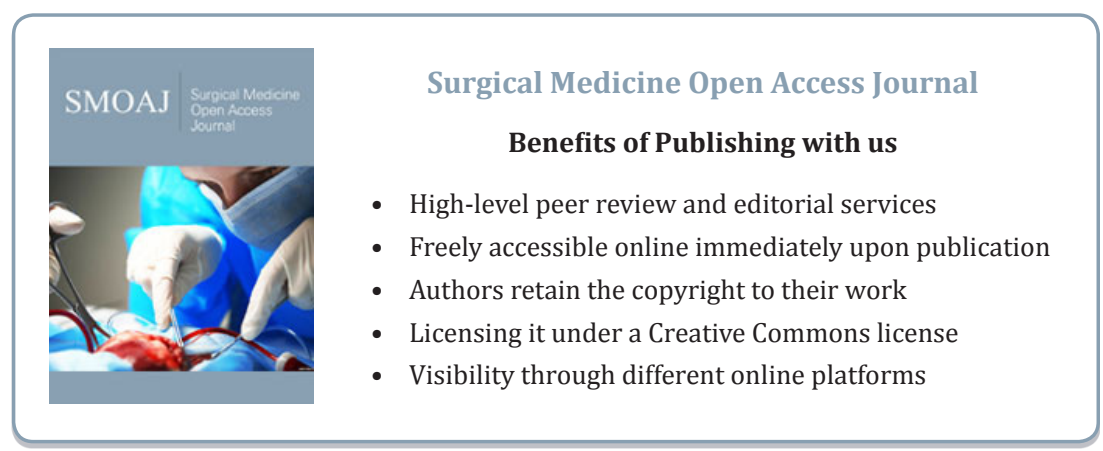

